\title{
¿Qué competencias científicas promovemos en el aula?
}

\author{
Guillermo Chona Duarte \\ Judith Arteta Vargas \\ Sonia Martínez \\ Ximena Ibáñez Córdoba \\ Marlén Pedraza* \\ Guillermo Fonseca Amaya**
}

Artículo recibido: 28-02-2005 y aprobado:28-09-2006

\section{What scientific competencies we promote in the classroom?}

Resumen: La educación en ciencias demanda en la actualidad la realización de procesos de formación integral en los estudiantes, que promuevan el desarrollo de competencias para enfrentar el mundo cambiante. En este artículo se muestran los resultados de los aspectos relacionados con los profesores participantes en la investigación "Competencias científicas y formación en valores. Un estudio desde el pensamiento de los profesores de ciencias experimentales", orientan en los alumnos a través de sus prácticas de aula.

Palabras clave: Competencias científicas, enseñanza de las ciencias experimentales, conocimiento profesional del profesor.
Abstract: The science education demands at the present time the accomplishment of processes of integral formation in the students, who promote the development of competencies to face the changing world. This article shows the results of the aspects related to the determination of the scientific competences that appear participant teachers in the investigation "Scientific competencies and formation in values. A study from the thought of the teachers of experimental sciences",orients in the students through their practices of classroom.

Key words: scientific competencies, education of experimental sciences, teachers' professional knowledge.

* Profesores Universidad Pedagógica Nacional, Grupo de Investigación biología, enseñanza y realidades. Participaron en la investigación, además de los autores, once profesores de cinco instituciones de educación básica y media de Bogotá. gchona@uni.pedagogica.edu.co

** Profesor catedrático Universidad Pedagógica Nacional,Grupo de Investigación Biología, Enseñanza y Realidades. Profesor tiempo completo proyecto curricular licenciatura en biología. Universidad Distrital Francisco José de Caldas.

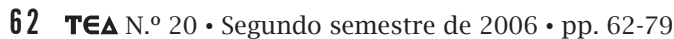




\section{Introducción}

En la última década, las políticas educativas colombianas han enfatizado en la necesidad de mejorar la calidad de la educación en el país (MEN, 1994, 1998, 2002). Es así como hoy podemos afirmar que se ha generado consenso en la comunidad educativa en general, en torno a que se requieren replanteamientos en el enfoque y tratamiento de la enseñanza y el aprendizaje de las áreas obligatorias y fundamentales, en concordancia con los fines propuestos para la educación en la Ley 115 de 1994, particularmente en las actuales condiciones de vida de la sociedad colombiana.

Aun cuando desde esta perspectiva se han realizado cambios en el enfoque y los contenidos curriculares, y se han sugerido múltiples estrategias metodológicas para abordar su trabajo en el aula, los cambios más polémicos han sido los implementados en el sistema de evaluación, tales como promoción automática, evaluación cualitativa, indicadores de logros y evaluación de competencias básicas.

El tema de las competencias surge dentro de las recomendaciones de la Misión de Ciencia, Educación y Desarrollo (1995), y ha sido introducido al medio educativo colombiano, con el fin de fortalecer el Sistema Nacional de Evaluación de la Educación, desde una perspectiva de mejoramiento de la calidad. Por lo anterior, es necesario contar con pautas o normas comunes, precisas y básicas que se establezcan como criterios que especifiquen lo que todos los estudiantes deben saber y ser capaces de hacer en los niveles preescolar, básico y medio (MEN, 2002).
Los resultados de exámenes de Estado y la existencia de documentos en los que se muestran variadas interpretaciones del orden teórico acerca del concepto de competencias (Cárdenas, 1999; Escobedo, 2001; Díaz, 2002) han generado en los maestros inquietudes respecto a reorientaciones para las áreas de enseñanza. Así, reflexionar sobre las prácticas pedagógicas en la enseñanza de las ciencias naturales con maestros en ejercicio, permite reconocer lo que éstos realizan desde su experiencia, donde sus discursos y prácticas son referentes para construir nuevas miradas en torno a los retos que implica ser partícipe en una sociedad dinámica y cambiante.

Lo anterior obliga a efectuar una revisión y un análisis conjunto con los profesores sobre nuevos enfoques hacia la calificación de la educación. En este contexto, en desarrollo de la investigación "Competencias científicas y formación en valores"” se indagó sobre las competencias científicas que once maestros de educación básica secundaria y media del Distrito Capital, promueven en sus estudiantes.

\section{El problema}

En investigaciones anteriores de los miembros de este grupo se han mostrado algunas dificultades que tienen los profesores de ciencias para incentivar la formación científica de un modo sistemático (Chona et al., 2001; Arteta et al., 2002). De igual manera, la sociedad colombiana le exige al sistema educativo

\footnotetext{
1 Investigación financiada por el Centro de Investigaciones de la Universidad Pedagógica Nacional, CIUP, y realizada en el periodo $2000-2003$.
} 
la formación de ciudadanos autónomos, conscientes, críticos y capaces de resolver problemas; esto es posible si los educadores están preparados para asumir tales retos.

Para el desarrollo de esta investigación nos preguntamos:

- ¿En qué queremos que sean competentes los estudiantes cuando aprenden ciencias naturales?

- ¿Qué competencias es posible desarrollar en los estudiantes a través de la enseñanza de las ciencias naturales?

\section{Referentes teóricos}

Sobre el concepto competencia hay diversidad de discursos teóricos: lingüístico (Chomsky, 1971), psicológico (Torrado, 1999), comunicativo (Hymes, 1972), laboral (Delors, 1996; Gómez, 1998), educativo (Gardner, 1993; Perkins, 1998), que en algunos casos se interrelacionan para generar una comprensión amplia; lo anterior sirve de base para afirmar que el término es polisémico y complejo; su introducción en el ámbito educativo implica no sólo revisar la evaluación y sus instrumentos formales de aplicación sino remirar lo educativo en general ligado con lo social, con lo cultural y con aspectos del aprendizaje y sus factores asociados. Por tanto, sin desconocer la complejidad de la conceptualización y que además la introducción del discurso sobre competencias en el medio educativo no es neutral porque tiene orígenes e implicaciones ideológicas, políticas y económicas, entre otras, el desarrollo de la investigación se circunscribió a la perspectiva educativa, reconociendo los aportes de los distintos enfoques.
El término competencia aparece citado en el documento sobre reforma al examen de Estado, definida así:

Competencia es un saber hacer en contexto, es decir, el conjunto de acciones que un estudiante realiza en un contexto particular y que cumplen con las exigencias específicas del mismo. En el examen de Estado las competencias se circunscribirán a las acciones de tipo interpretativo, argumentativo y propositivo que el estudiante pone en juego en cada uno de los contextos disciplinares que hacen referencia, por su parte, al conjunto móvil de conceptos, teorías, historia epistemológica, ámbitos y ejes articuladores, reglas de acción y procedimientos específicos que corresponden a un área determinada (MEN-Icfes, 1999, pp. 10-11).

En varios artículos de Cárdenas (1998, 2000), y de Cárdenas y Sarmiento (2000), se hace el planteamiento general sobre la importancia de la educación en ciencias para el desarrollo humano y económico; ésta, según los autores, es un medio de humanización en que se reconoce la necesidad de que la juventud adquiera y practique ciertos conocimientos, habilidades intelectuales, valores y perspectivas científicas.

La habilidad intelectual, siguiendo a Cárdenas (1998), es el talento, capacidad y disposición para la comprensión de los conceptos y procedimientos científicos:

... la educación en ciencias propende al desarrollo de las siguientes capacidades a lo largo de la vida escolar de los alumnos: habilidades básicas, habilidades de procedimiento y habilidades investigativas. Igualmente, a través de la enseñanza de las ciencias es posible desarrollar algunas habili- 
dades, propias del dominio afectivo, como la capacidad de emitir juicios de valor, el respeto por la forma de pensar de los demás, la capacidad del trabajo en grupo, la tolerancia y la convivencia social" (Cárdenas, 1998, p.57).

Adicionalmente, Cárdenas (1998) reconoce dos clasificaciones en relación con las competencias en ciencias:

- La propuesta por el Comité de Calidad de la Educación en Australia en 1985, donde se establecen como competencias: "Adquisición de información, comunicación de la misma, aplicación de procesos lógicos, desarrollo de tareas prácticas y tareas en grupo" (Cárdenas, 1998, p.59).

- La propuesta por el Mayer Committee-Melbourne 1992, que establece las siguientes competencias: recolección, análisis y organización de información, comunicación de información e ideas; planeación y organización de actividades; capacidad de trabajo en grupo; empleo de ideas y técnicas matemáticas; resolución de problemas y uso de la tecnología.

Cárdenas y Sarmiento (2000) establecen tres niveles de competencia deseables para los estudiantes de educación básica y media:

- El primero, en relación con "el reconocimiento y distinción del sistema básico de significación, que se encuentra asociado a los procesos de abstracción, conceptualización, y simbolización que realizan los seres humanos" (Cárdenas y Sarmiento, 2000, p. 201). En él se reúnen los códigos y formas de comunicación particular de una disciplina.

- El segundo nivel hace referencia "a la interpretación y uso comprensivo; este nivel está asociado con el establecimiento de relaciones entre conceptos, el uso correcto de la mediación y el razonamiento lógico, lo cual permite la apropiación de un cuerpo de conocimientos básicos acerca de los temas estudiados. En este nivel de competencia el estudiante reconoce y utiliza las nociones aprendidas en distintos contextos". (Cárdenas y Sarmiento, 2000, p.202).

- El nivel tres, argumentación y síntesis, “... se manifiesta en el ejercicio de la intuición y la creatividad. El estudiante avanza más allá del conocimiento aprendido intuyendo e imaginando otras posibilidades de realización o explicación" (Cárdenas y Sarmiento, 2000, pp. 202-203).

Escobedo (2001, p. 47), establece que “... desde la perspectiva de la educación para ser competentes en un determinado campo, es necesario conocer y comprender, poder cooperar armónicamente con los demás, ser sensible a los problemas del campo y sentir gusto en trabajar para tratar de resolverlos". En esta aproximación se reconoce una cualidad de la competencia y es su utilidad en la resolución de una tarea o problema.

Respecto a cómo ser competente en ciencias naturales, Escobedo señala que “... una persona es competente para ser productiva en las ciencias naturales cuando ha logrado desarrollar el pensamiento científico, desarrollar la capacidad de trabajar en equipo y desarrollar el interés por el conocimiento científico" (Escobedo, 2001, p. 47). El autor interpreta la competencia científica como una unidad donde el pensamiento científico se entiende como la capacidad de comprender los procesos de lo real, 
manejar el lenguaje de la ciencia de manera oral y escrita, dominar el lenguaje especializado de la ciencia, criticar las teorías de los demás y las propias, conocer sobre la forma como uno conoce. Ello se complementa con el trabajo en equipo, que implica escuchar y entender los argumentos de los demás, saber reconocer los aspectos positivos y negativos del trabajo del otro desde el respeto, hacer observaciones interesantes que permitan avanzar en el conocimiento científico; estos aspectos se conjugan con un tercer elemento de la unidad, el interés por el conocimiento científico, que definitivamente es relevante ya que en él se encuentra el componente afectivo por la ciencia.

\section{Competencias científicas en la presente investigación}

En esta investigación se trabajan las competencias desde la perspectiva educativa, dado que nos interesa hacer las lecturas y proyecciones que los profesores de ciencias experimentales participantes en este estudio realizan del discurso sobre competencias que circula en el medio educativo colombiano; en general, en la política evaluativa actual, y en particular, su implementación en la enseñanza y el aprendizaje del área de ciencias naturales y educación ambiental.

Por lo anterior, basados en los referentes teóricos consultados y en las reinterpretaciones y reconstrucciones conceptuales surgidas en las discusiones con los maestros participantes, para la presente investigación se conceptualiza competencia científica como la capacidad de un sujeto, expresada en desempeños observables y evaluables que evidencia formas sistemáticas de razonar y explicar el mundo natural y social, a través de la construcción de interpretaciones apoyados por los conceptos de las ciencias. Se caracteriza por la movilidad y flexibilidad en el tiempo y en el espacio, posibilitando que el sujeto en su actuación muestre las actitudes, principios y procedimientos propios de la ciencia. Así, "las competencias científicas se desarrollan en la interrelación de los contextos disciplinar, multicultural y de la vida cotidiana" (Arteta et al, 2002, p. 274).

A partir de los hallazgos de la investigación, las competencias científicas se categorizaron como básicas, investigativas y de pensamiento reflexivo $y$ crítico, en niveles inicial, intermedio $y$ avanzado.

Las competencias científicas básicas incluyen la capacidad de un sujeto para reconocer un lenguaje científico, desarrollar habilidades de carácter experimental, organizar información y trabajar en grupo.

El reconocimiento de un lenguaje científico se emplea para denotar las características o propiedades de un fenómeno, el cual permite establecer procesos de comunicación a través de un código relativo al campo de las ciencias experimentales y dentro de su comunidad.

El desarrollo de habilidades de carácter experimental hace referencia principalmente a la manipulación de material de laboratorio, lo cual permite el uso apropiado de instrumentos a partir de seguimiento de instrucciones y la ejecución de algunas tareas sencillas en relación con habilidades procedimentales propias de las ciencias. 
La organización de la información propicia la capacidad de interpretar, clasificar y presentar, mediante distintas formas como textos, tablas, gráficas, diagramas, dibujos y esquemas, datos e ideas en relación con características de objetos, eventos y fenómenos naturales, haciendo posible su comunicación e interacción con los otros.

El trabajo en grupo se entiende desde la posibilidad que tienen los sujetos de confrontar sus ideas, establecer acuerdos y desarrollar tareas de una manera conjunta.

Las competencias científicas investigativas se asumen como la capacidad del sujeto de construir explicaciones y comprensiones de la naturaleza desde la indagación, la experimentación y la contrastación teórica, donde se formula un problema genuino que le genera conflicto cognitivo y desde un trabajo sistemático interrelaciona conceptos con los cuales establece argumentaciones que dan cuenta de los fenómenos naturales.

El aspecto experimental dentro de esta competencia incluye las posibilidades de reconocer elementos constitutivos de la experimentación y también asociarlas a la resolución de problemas a partir de otro tipo de experiencias de aprendizaje, como la confrontación conceptual a través de diferentes fuentes de información. Incluye, además, la socialización en la presentación de los resultados, lo cual permite la construcción individual y colectiva de conocimiento por medio de los espacios de discusión que se generan.

Los problemas que se abordan pueden ser de carácter disciplinar o cotidiano, y respecto a su origen, formulados por el docente, por los estudiantes o conjuntamente.

Las competencias de pensamiento reflexivo y crítico se entienden como la capacidad que tiene un sujeto de desarrollar procesos cognitivos que van más allá de la selección y procesamiento de la información, permitiéndole integrar creativa y propositivamente los saberes frente a nuevas situaciones, resolviendo problemas desde una postura crítica, ética y de construcción de significados contextualizados.

Con respecto a los procesos cognitivos, (Cárdenas, 1998), se consideran entre otros la seriación, clasificación, discriminación, análisis y síntesis, que no se desarrollan en abstracto sino a través de las mismas actividades de clase en relación con un objeto de estudio. La postura crítica implica que, además de comprender los fenómenos, se construya una mirada particular en torno a la ciencia misma y sus implicaciones en la sociedad, reconociéndose como un sujeto dentro del mundo natural y social. La construcción de significados está ligada al reconocimiento, contrastación e interpretación que hace el sujeto de diversas fuentes de información, elaborando su propio universo de comprensión sobre los eventos y fenómenos naturales.

\section{Pensamiento del profesor y} conocimiento profesional del profesor Por otra parte, en esta investigación -teniendo en cuenta la función fundamental del profesor en el desarrollo de competencias en los estudiantes- se acogen los planteamientos surgidos de la línea de investigación conocida internacionalmente como pensamiento del profesor, con base en los hallazgos 
de anteriores investigaciones del grupo que sirven de antecedentes (Chona et al., 1998 y 2001; Reyes et al., 1999).

Pérez y Gimeno (1988) muestran que existen dos enfoques principales en la investigación sobre el pensamiento del profesor: uno, eminentemente psicologista, preocupado por discriminar los procesos formales de procesamiento de la información, y otro, pedagógico, centrado en los contenidos, ideas y teorías del profesor sobre los procesos de enseñanza y aprendizaje. La presente investigación se ubica más en esta última perspectiva.

Siguiendo este último enfoque, Pérez y Gimeno consideran que el conocimiento del profesor es "tácito, poco articulado y organizado con una lógica peculiar, que responde más a las prioridades afectivas establecidas en la historia personal-profesional" (Pérez y Gimeno, 1988. En Gallego, 1991, p.290), que a las teorías pedagógicas. De igual manera, otros autores ponen en evidencia que las creencias de los profesores de ciencias acerca de su práctica y del aprendizaje de los estudiantes están más relacionadas con su sistema de concepciones, que con los mandatos de los nuevos currículos (Yerrit et al., 1997).

En el análisis de la relación pensamiento- acción del profesor, las influencias del conocimiento, creencias, valores y destrezas desempeñan un papel preponderante, aun en fases iniciales de su formación. "Los profesores, al igual que otras personas, orientan su conducta a partir del conocimiento y creencias que poseen. Y este conocimiento y creencias se empiezan a construir mucho antes que el profesor decida dedicarse profesionalmente a la enseñanza" (Marcelo,
2002, p. 47. En Perafán y Adúriz-Bravo, 2002).

Las creencias influyen en la forma como se caracterizan los acontecimientos y en cómo estimamos la variación simultánea de fenómenos; incluso la contrastación de nuestras creencias con la experiencia está influida por las propias creencias y por su interacción con la manera como percibimos los datos.

Los profesores, sobre todo los de más experiencia, tienen normas y principios de actuación en la práctica que guían su planificación y su conducta interactiva (Reyes y Salcedo, 1998).

Es importante señalar que en torno al conocimiento profesional se han desarrollado investigaciones que lo caracterizan; así, se destacan los trabajos de Shulman (1989), Porlán (1997) y Marcelo (2002). Al respecto, Shulman (1989) aborda el conocimiento del profesor desde la perspectiva del cononocimiento del contenido, clasificándolo en tres clases: la materia que se va a enseñar, el pedagógico y el curricular, mientras que años después Porlán (1997, p. 158) propone que el conocimiento profesional del profesor es "el resultado de yuxtaponer cuatro tipos de saberes de naturaleza diferente, generados en momentos y contextos no siempre coincidentes, que se mantienen relativamente aislados unos de otros en la memoria de los sujetos y que se manifiestan en distintos tipos de situaciones profesionales o preprofesionales". Estos cuatro saberes son el saber académico, los saberes basados en la experiencia, las rutinas y guiones de acción y las teorías implícitas.

Marcelo (2002) propone integrar tres tipos de conocimiento: el psicopedagógico, el conocimiento del contenido y el 
conocimiento didáctico del contenido. Además, señala tres aspectos importantes respecto al conocimiento del profesor, que se construye en la interacción social, de carácter contextualizado, y está distribuido entre grupos y ambientes simbólicos y físicos.

\section{Metodología}

La investigación se orientó desde los presupuestos del paradigma interpretativo, el cual, como lo plantea Wittrock (1997), ofrece la posibilidad de comprender la realidad del maestro en el aula, con participación de los sujetos involucrados en las propias acciones estudiadas, brindando la opción de construir conocimientos, en los cuales, desde una perspectiva hermenéutica (Carr y Kemmis, 1988), permite interpretar las realidades escolares más allá de lo meramente instrumental y normativo.

Desde este paradigma, la investigación siguió la modalidad de estudio de casos múltiples, acogiendo la tipología propuesta por Arnal et al. (1992, p. 206), quienes plantean que el estudio de caso "es la forma más propia y característica de las investigaciones ideográficas llevadas a cabo desde la perspectiva cualitativa...". Se caracteriza por "su capacidad para generar hipótesis y descubrimientos, y en centrar su interés en un individuo, evento o institución y en su flexibilidad y aplicabilidad a situaciones naturales". La base para el estudio de caso son la descripción y el análisis detallado de las unidades definidas para el estudio, con lo cual se busca comprender la situación analizada.

El estudio se realizó en cinco instituciones de la ciudad de Bogotá, con la participación de once maestros, cada uno de los cuales se constituyó y analizó como un caso. El trabajo de campo incluyó el diseño y la ejecución de seminarios y talleres, con la participación de los profesores junto con el equipo de investigación; la observación y filmación de tres ambientes de clase de cada profesor, el desarrollo de entrevistas, así como el trabajo de discusión y producción de interpretación colectiva propios de este tipo de investigación.

Con base en la construcción conjunta con los profesores, a través de su participación en conversatorios, talleres y debates, que se analizaron a la luz de referentes teóricos, se elaboró y aplicó un instrumento (Chona et al., 2004) para la lectura, interpretación y categorización de las competencias clasificadas como básicas, investigativas y de pensamiento reflexivo y crítico. La interpretación de cada caso permitió proponer orientaciones didácticas para el desarrollo de competencias científicas en el aula. En el esquema del gráfico 1 se presenta una síntesis de la metodología.

\section{Resultados y discusión}

En la tabla 1 se presentan los 65 desempeños de las categorías y niveles de competencias científicas que se definieron en la metodología. En la columna final se muestra la frecuencia con que se evidenciaron en las clases de los once profesores estudiados.

\section{Competencias científicas básicas}

Con respecto a las competencias básicas del nivel inicial, todos los docentes orientan en sus estudiantes los desempeños 1, 4 y 9. El desempeño 1 se expresa en las actividades realizadas y 


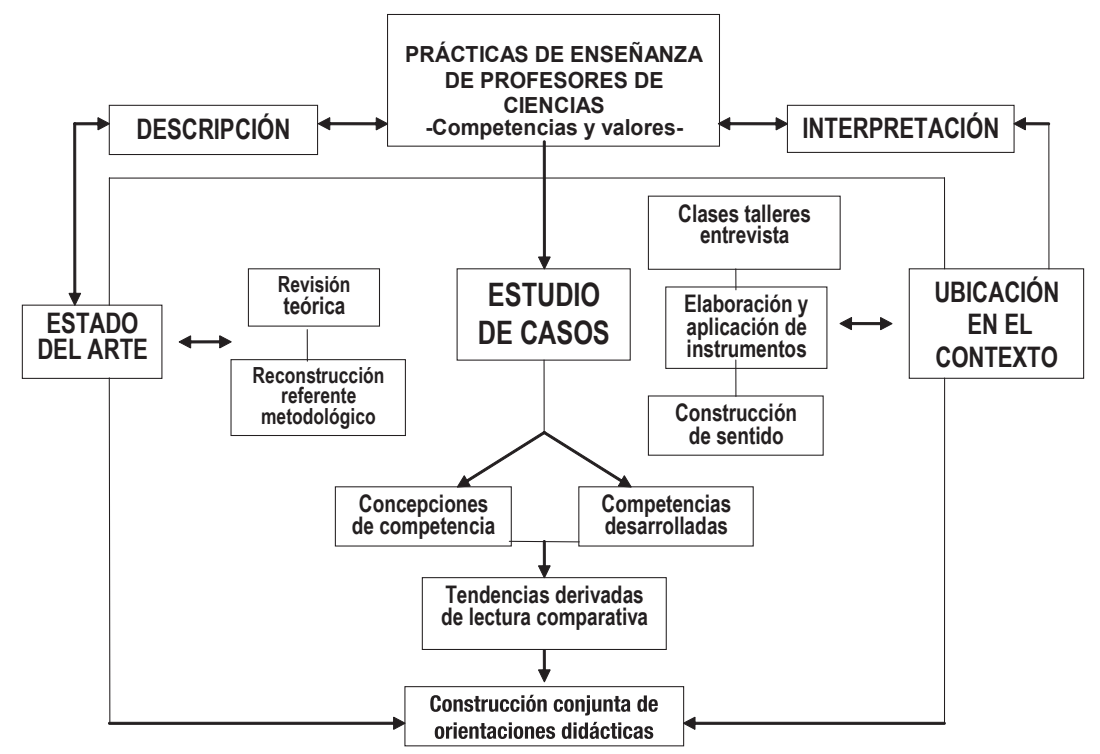

Gráfico 1. Proceso metodológico cualitativo.

cobra sentido en la medida en que se percibe en los profesores un especial interés por desarrollar en los alumnos la capacidad para describir su entorno o diferentes fenómenos, donde la exploración es elemento fundamental dentro de los procesos de aprendizaje de los estudiantes.

En torno a este desempeño se identifican dos tendencias: una asociada a la observación y descripción directa de objetos y fenómenos, en estrecha relación con trabajos de orden experimental, y otra que prevalece en la mayor parte de los casos, asociada con la descripción de las representaciones que tienen los estudiantes sobre los fenómenos.

Vale la pena mencionar que algunos docentes trabajan los objetos de estudio en una perspectiva de construcción de conocimiento, donde la información no es el fin del proceso de enseñanza, sino un elemento importante en la comprensión de éste. Otros docentes propician la observación del objeto, evento o fenómeno desde las preguntas que guían la indagación sobre el mismo, confrontando y validando la información que existe sobre el objeto. La observación para los profesores permite, en primera instancia, describir y explicar, aunque no necesariamente tiene correspondencia con la aplicación del método científico en la enseñanza de las ciencias.

En cuanto a la capacidad para seguir instrucciones (desempeño 4), por una parte, se centra en ofrecer instrucciones donde el éxito de las actividades está mediado por la atención de los estudiantes y la puesta en marcha de las orientaciones dadas; por otro lado, este desempeño se puede interpretar como una de las características del quehacer científico que es importante propiciar 
en el marco de la formación en ciencias experimentales.

En este último caso, tal desempeño podría pensarse en dos vías de interpretación: la primera tiene que ver con la importancia de seguir instrucciones para posibilitar en el estudiante la reflexión sobre la trascendencia de la secuencialidad y el orden y por otra parte, alcanzar resultados desde un ejercicio operativo.

La capacidad para trabajar en grupo es un aspecto que hay que destacar por el privilegio que se ha venido otorgando a la labor en equipo como estrategia impulsada en las últimas reformas edu- cativas y enfoques didácticos. Todos los docentes propician en sus estudiantes la capacidad para trabajar en grupo (desempeño 9); para algunos, este desempeño se constituye en un espacio de verdadera construcción conjunta, donde cobran significado la interacción, la discusión y los ejercicios de confrontación conceptual propios del quehacer científico y de la enseñanza dialógica. Sin embargo, nos surge la inquietud frente a cierto trabajo grupal que corresponde más a un ejercicio de ubicación espacial y de reunión de estudiantes que realmente no trasciende hacia un verdadero intercambio de ideas en todo el grupo.

Tabla 1. Competencias y desempeños orientados por los profesores y observables en los estudiantes

\begin{tabular}{|c|c|c|c|c|}
\hline 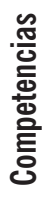 & $\stackrel{\mathscr{\Phi}}{\Phi}$ & \multicolumn{2}{|r|}{ Desempeños } & \multirow{2}{*}{$\begin{array}{l}\text { 즁 } \\
\text { 으 } \\
11\end{array}$} \\
\hline \multirow{25}{*}{ 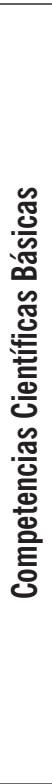 } & \multirow{9}{*}{ 可 } & 1 & Capacidad para observar y describir objetos, eventos o fenómenos & \\
\hline & & 2 & Manipular instrumentos de medida & 2 \\
\hline & & 3 & Habilidad para realizar mediciones de diferentes magnitudes & 1 \\
\hline & & 4 & Capacidad para seguir instrucciones & 11 \\
\hline & & 5 & Habilidad para recolectar datos & 6 \\
\hline & & 6 & Capacidad para comunicar información e ideas de manera oral y escrita & 10 \\
\hline & & 7 & Reconocer y emplear un lenguaje científico & 10 \\
\hline & & 8 & Capacidad para interpretar gráficas que describen eventos & 3 \\
\hline & & 9 & Capacidad para trabajar en grupo & 11 \\
\hline & \multirow{12}{*}{$\begin{array}{l}\text { 응 } \\
\text { 튼 } \\
\text { 을 }\end{array}$} & 10 & $\begin{array}{l}\text { Capacidad para observar, describir y establecer relaciones entre las } \\
\text { características de objetos, eventos o fenómenos en distintos contextos }\end{array}$ & 9 \\
\hline & & 11 & Calcular valores que involucren dos variables (densidad, molaridad, etc.) & 4 \\
\hline & & 12 & Recolectar datos y organizar información mediante tablas y gráficas & 3 \\
\hline & & 13 & Capacidad para combinar ideas en la construcción de textos & 6 \\
\hline & & 14 & Identificar el esquema ilustrativo correspondiente a una situación & 2 \\
\hline & & 15 & $\begin{array}{l}\text { Identificar la gráfica que relaciona adecuadamente dos o más variables que } \\
\text { describen el estado, las interacciones o la dinámica de un evento }\end{array}$ & 2 \\
\hline & & 16 & Interpretar y construir gráficas & 2 \\
\hline & & 17 & Planear y organizar actividades & 8 \\
\hline & & 18 & Emplear ideas y técnicas matemáticas & 3 \\
\hline & & 19 & Utilizar recursos tecnológicos & 1 \\
\hline & & 20 & Comprender y escribir textos científicos & 3 \\
\hline & & 21 & Utilizar material de laboratorio & 2 \\
\hline & \multirow{4}{*}{$\begin{array}{l}\text { 응 } \\
\mathbb{N} \\
\text { 저 } \\
\text { Z }\end{array}$} & 22 & $\begin{array}{l}\text { Generalizar y extender determinados conceptos o propiedades a un dominio más } \\
\text { amplio o en distintos contextos }\end{array}$ & 7 \\
\hline & & 23 & Generar nuevos desarrollos conceptuales & 3 \\
\hline & & 24 & $\begin{array}{l}\text { Plantear relaciones condicionales para que un evento pueda ocurrir o predecir lo } \\
\text { que pueda suceder, dadas las condiciones sobre ciertas variables. }\end{array}$ & 3 \\
\hline & & 25 & Resolver problemas de lápiz y papel que involucren dos o más variables & 4 \\
\hline
\end{tabular}


Tecné, Episteme y Didaxis N. ${ }^{\circ} 20,2006$

\begin{tabular}{|c|c|c|c|c|}
\hline \multirow{12}{*}{ 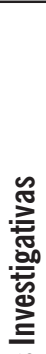 } & \multirow{10}{*}{ 可 } & 26 & Formular preguntas sobre hechos o fenómenos & 7 \\
\hline & & 27 & Proponer posibles explicaciones & 9 \\
\hline & & 28 & Identificar los diseños experimentales pertinentes para contrastar hipótesis & 2 \\
\hline & & 29 & Establecer variables & 1 \\
\hline & & 30 & $\begin{array}{l}\text { Utilizar selectivamente la información para interpretar e interactuar en forma } \\
\text { adecuada }\end{array}$ & 10 \\
\hline & & 31 & Presentar la información a través de textos & 4 \\
\hline & & 32 & Resolver problemas sencillos & 8 \\
\hline & & 33 & Proponer o establecer los procedimientos para abordar problemas & 4 \\
\hline & & 34 & Buscar información pertinente para ilustrar una situación problemática & 6 \\
\hline & & 35 & Desarrollo de pensamiento causal & 10 \\
\hline & \multirow{9}{*}{$\begin{array}{l}\text { 응 } \\
\text { 틀 } \\
\text { 튼 }\end{array}$} & 36 & Formular preguntas sobre hechos o fenómenos derivados de la experimentación & 3 \\
\hline & & 37 & Formular y contrastar hipótesis & 3 \\
\hline \multirow{15}{*}{ 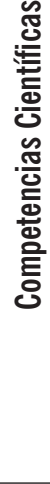 } & & 38 & Predecir los resultados de un proceso & 2 \\
\hline & & 39 & Diseñar experimentos para contrastar una hipótesis & 1 \\
\hline & & 40 & Establecer relaciones entre variables & 2 \\
\hline & & 41 & Seleccionar procedimientos e instrumentos adecuados & 3 \\
\hline & & 42 & Presentar la información a través de tablas, gráficas, diagramas y esquemas & 5 \\
\hline & & 43 & Emplear ideas y técnicas matemáticas & 2 \\
\hline & & 44 & Utilizar la argumentación en la discusión de sus ideas & 9 \\
\hline & \multirow{8}{*}{$\begin{array}{l}\text { 응 } \\
\text { N } \\
\text { ত্ত } \\
\text { Z }\end{array}$} & 45 & $\begin{array}{l}\text { Simbolizar los conceptos y utilizarlos en la construcción de explicaciones y su } \\
\text { matematización }\end{array}$ & 4 \\
\hline & & 46 & Plantear problemas de tipo contextual o disciplinar & 7 \\
\hline & & 47 & $\begin{array}{l}\text { Concebir formas alternativas de explicación a una situación dada, a partir del } \\
\text { manejo de procedimientos y conceptos científicos }\end{array}$ & 3 \\
\hline & & 48 & $\begin{array}{l}\text { Diseñar y poner a prueba montajes experimentales o procedimientos para } \\
\text { contrastar hipótesis }\end{array}$ & 1 \\
\hline & & 49 & Predecir situaciones en las que intervengan diversas condiciones sobre variables & $\mathbf{0}$ \\
\hline & & 50 & Generalizar a partir de observaciones & 4 \\
\hline & & 51 & Presentar la información a través de modelos explicativos & 5 \\
\hline & & 52 & Resolver problemas disciplinares o derivados de la cotidianidad & 7 \\
\hline \multirow{13}{*}{ 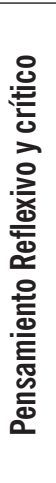 } & \multirow{2}{*}{ 홍 } & 53 & $\begin{array}{l}\text { Habilidades de pensamiento: clasificación, seriación, discriminación, sintesis y } \\
\text { discusión interactiva }\end{array}$ & 7 \\
\hline & & 54 & Razonamiento lógico & 9 \\
\hline & \multirow{4}{*}{$\begin{array}{l}\text { 응어 } \\
\text { 튼 } \\
\text { 트 }\end{array}$} & 55 & Tomar decisiones, en la selección de información, datos o procedimientos & 8 \\
\hline & & 56 & Construir y reconstruir significados a partir de la información consultada & 6 \\
\hline & & 57 & Emplear conceptos matemáticos para la construcción de significados & 3 \\
\hline & & 58 & Hacer inferencias & 6 \\
\hline & \multirow{7}{*}{$\begin{array}{l}\text { 응 } \\
\mathbb{N} \\
\text { ত̃ } \\
\text { 전 }\end{array}$} & 59 & Utilizar pensamiento critico y creativo en la solución de problemas & 3 \\
\hline & & 60 & Emplear pensamiento causal y multicausal & 8 \\
\hline & & 61 & Resolver problemas disciplinares o cotidianos desde los principios de la ciencia & 6 \\
\hline & & 62 & Ser coherente entre su pensamiento y acción & 2 \\
\hline & & 63 & Elaborar teorias explicativas & 2 \\
\hline & & 64 & Asumir con sentido critico y ético el trabajo cientifico & 3 \\
\hline & & 65 & Respetar la dinámica propia de la naturaleza y reconocerse en ella & 2 \\
\hline
\end{tabular}

Todos los profesores participantes en esta investigación consideran fundamental el trabajo grupal, bien sea desde la convivencia, la organización, la importancia de asumir responsabilidades y compromisos, o como espacio de debate y contrastación conceptual.

La mayoría de los docentes promueven los desempeños 6 y 7, considerando que una de las características de la ciencia es el empleo de un sistema de significación básico, que se usa como lenguaje para representar diferentes fenómenos y propiedades de lo que se estudia. Así mismo, desarrollar en los estudiantes habilidades comunicativas se constituye en un aspecto de especial importancia, no sólo para una formación en ciencias, sino en general para la formación de ciudadanos que posean los elementos básicos para desenvolverse en un medio social. 
Respecto al desempeño 6, cabe señalar que en algunos docentes se evidencia la relación entre pensamiento y lenguaje; en este sentido, el uso comprensivo del lenguaje científico genera una estrecha relación con el pensamiento científico, donde el estudiante desarrolla un ejercicio racional que incluye procesos como formulación de hipótesis, argumentación y, en general, explicaciones respecto a los fenómenos naturales.

En relación con el desempeño 7, en el grupo de profesores que lo propician se encontraron dos aspectos esenciales: el primero, asociado a la apropiación de los códigos lingüísticos propios del campo de las ciencias naturales, donde el estudiante a través de un lenguaje especializado establece una coherencia entre su pensamiento y acción, lo que se permite la comprensión misma del fenómeno que desea explicar; es decir, el acto comunicativo es inteligible para quienes participan.

Por otra parte, en algunas de las clases circulan diversos códigos de comunicación entre los estudiantes y el maestro, y aunque se enuncian palabras propias del campo de las ciencias naturales, tal acción no garantiza la comprensión de las mismas, en tanto que no se genera con los estudiantes un espacio de discusión respecto a su significado; así, parece que las palabras debieran significar por si mismas. Vale la pena anotar que algunos de los profesores hacen referencia a la importancia del empleo adecuado de la terminología científica, no solamente en el contexto de las clases, sino en la vida cotidiana, aspecto que en realidad puede reflejar la apropiación y comprensión del lenguaje científico por parte de los estudiantes.
Así mismo, los docentes poco propician los desempeños 5 y 8 , en tanto que escasos docentes hacen énfasis en los desempeños 2 y 3, los cuales están asociados con actividades de carácter experimental. Estos resultados se constituyen en un indicador de la necesidad de incentivar a los profesores para propiciar más este tipo de desempeños en los estudiantes, teniendo en cuenta el papel que cumplen la manipulación de instrumentos y la medición en relación con el carácter experimental de las ciencias.

Así, pocos maestros reconocen en el estudiante un sujeto que aprende desde la interacción con un objeto de conocimiento, que lo manipula, lo intenta explicar desde el reconocimiento de las propiedades del objeto y desde la interacción de éste con otros; el desarrollo de tales desempeños puede acompañarse de la incertidumbre, ya que es la multiplicidad de interpretaciones sobre el objeto lo que genera procesos de confrontación y construcción conceptual.

Adicionalmente, cabe precisar que en las en las clases en las que se evidenciaron estos desempeños se abordaron temáticas de química donde se planea experimentación por parte de los estudiantes, mientras que en las clases de biología se continúa desde una perspectiva más descriptiva.

Con respecto a las competencias básicas del nivel intermedio, se destacan los desempeños 10, 17 y 13, con las más altas frecuencias. En estos desempeños hay que resaltar que en una relación dialógica entre estudiante y maestro se involucra el contexto como parte del objeto de estudio y en algunos casos se inician procesos de transformación del 
mismo; la enseñanza toma en cuenta el contexto cotidiano, el cual se comprende y explica desde diversas fuentes de información. El reconocimiento del contexto genera en algunos casos el desarrollo de metodologías, donde el estudiante es reconocido como sujeto de saber, que participa en procesos de transformación de su propia realidad.

Igualmente entre otros de los desempeños destacados con un alto número de profesores se encuentran el $17 \mathrm{y}$ el 13. En cuanto al primer aspecto, la posibilidad de diseñar actividades abre un espacio a la creatividad de los estudiantes, incentivándolos a ser organizados y estar pendientes del desarrollo de las acciones propuestas.

Solamente dos de los profesores orientan el desempeño 21, lo cual es un indicador de la necesidad de propiciar el carácter experimental de las ciencias y el papel que esto debería cumplir en los ámbitos y espacios de formación.

Con respecto a los desempeños 11 y 18 , los cuales presentan bajas frecuencias, cabe destacar que los propician únicamente aquellos docentes que trabajan en sus clases temáticas propias de la química o la física. De esta manera, el calcular valores que involucren dos variables y el emplear ideas y técnicas matemáticas se constituyen en desempeños característicos de las disciplinas mencionadas; no obstante tomando en cuenta el carácter común de las ciencias experimentales, cobra sentido reflexionar acerca de por qué dichos desempeños usualmente no son posibilitados, por ejemplo, al abordar temáticas de biología y educación ambiental.

Los desempeños 12, 14, 15 y 16 son promovidos por pocos docentes; en con- traposición, la mayoría de los profesores enfatiza en actividades como exposiciones, talleres sobre lecturas y trabajo grupal con base en guías. Apenas uno de los profesores observados involucra la utilización de recursos tecnológicos; dicha situación podría verse influenciada por la escasez y la baja disponibilidad de este tipo de materiales en las instituciones educativas.

Con respecto a las competencias básicas de nivel avanzado, se presenta con mayor frecuencia el desempeño 22; los otros tres desempeños que integran este grupo los propician pocos profesores.

\section{Competencias científicas investigativas}

En el presente estudio la competencia investigativa agrupa 27 desempeños (26 a 52), de los cuales la mayoría de los maestros desarrolla apenas ocho; este hallazgo nos permite reflexionar acerca de la perspectiva de enseñanza de la ciencia con que se forma a los estudiantes, quienes tienen que enfrentar las exigencias de un mundo contemporáneo que les exige competencias de este nivel.

En cuanto a los desempeños del nivel inicial, se observan mayores frecuencias en los desempeños 27, 30 y 35, mientras que los desempeños 28 y 29 presentan las más bajas frecuencias.

El desempeño 30, utilizar selectivamente la información para interpretar e interactuar en forma adecuada, es común en todas las clases; sin embargo, cabe señalar que la información que se privilegia es la voz del maestro y el texto escolar; en muy pocos casos se observa la posibilidad de emplear otras fuentes de información, como la voz de 
expertos, el periódico local, artículos científicos, otras posturas respecto a la temática propia. En el aula circula información que poco se conecta con preocupaciones reales de los estudiantes; en este sentido, no se garantiza que la información nueva genere un proceso de relación con sus preguntas e inquietudes. Así, en el aula de clases de ciencias todavía se privilegia la información y no la construcción conceptual significativa.

En la clase de ciencias se desarrolla una lógica de comprensión del mundo desde la causa-efecto. Con respecto al desempeño 35, diez de los once profesores generan preguntas del tipo ¿por qué sucede esto?, ¿a qué se debe...? Y afirmaciones que permiten afianzar esta lógica.

El cuanto al desempeño 36, se observó en siete de los docentes lo que significa que en la clase se generen espacios para que los estudiantes puedan interrogar hechos o fenómenos que les suceden a su alrededor. Así, en el desarrollo de las clases se pueden caracterizar dos tipos de preguntas por parte de los estudiantes: preguntas de tipo conceptual, como “¿Qué relación existe entre mitosis y genética y lo que hemos tenido durante todo el año?" y preguntas que se resuelven de manera inmediata y cuya respuesta el maestro orienta desde lo establecido en el contenido curricular. Sin embargo, otros estudiantes trascienden este tipo de interrogantes y formulan preguntasproblema, que ameritan un trabajo de consulta e investigación rigurosa, ya que la respuesta puede ser múltiple y compleja, por lo que deberá construirse con diversos elementos.
En los registros de las clases el desempeño 28 es poco observable, razón por lo cual merece que se reflexione sobre su escaso trabajo en las clases de ciencias naturales. En este sentido, se puede inferir que los desempeños que los maestros desarrollan en los estudiantes se relacionan más con la competencia teórica explicativa, coherente con el tipo de actividades que el maestro desarrolla en el aula. En relación con este desempeño se pueden asignar dos significados: el primero, que el diseño experimental se relaciona de manera complementaria con un trabajo de clase, lo que permite que el estudiante haga una confrontación entre lo que ha leído, ha comprendido y cómo este saber se evidencia en un ejercicio de tipo experimental. El segundo corresponde a unas prácticas de laboratorio que obedecen más a un ejercicio de repetición de procedimientos estandarizados, donde lo que se pretende es un ejercicio de emulación respecto al trabajo de los científicos y no al desarrollo de este tipo de desempeños en el propio estudiante.

El desempeño 29 se observó en las clases de un docente. Cabe señalar que para el caso de Colombia, los resultados de la pruebas de Estado muestran dificultades en los estudiantes en relación con estos desempeños, que es necesario atender.

En cuanto a los desempeños del nivel intermedio, sobresale el 44 , asociado a la capacidad de los estudiantes de dar explicaciones frente a los fenómenos abordados desde sus referentes conceptuales y vivenciales previos y al empleo de la información abordada en clase, derivada de la consulta y de las explicaciones de los profesores. 
Con respecto al desempeño 43, se evidencia en las clases de los maestros cuya temática se relaciona con la química, donde los estudiantes desarrollan ejercicios de lápiz y papel en relación con elementos matemáticos; con todo, en las clases observadas de biología no se evidencia este desempeño. Una posible hipótesis plantearía que la biología se asume desde lo descriptivo y no explicativo, como sucede en las áreas de física y química.

En general, los otros desempeños asociados a este nivel de competencia presentan bajas frecuencias. Cabe anotar que en este nivel los desempeños hacen referencia al desarrollo de habilidades derivadas de la experimentación, lo cual, como se expresó anteriormente, es un aspecto poco trabajado por los profesores en sus clases.

De igual manera, en relación con los desempeños del nivel avanzado se destacan con altas frecuencias los desempeños 46 y 52. Con respecto a ello, en muchos casos los problemas se asocian a ejercicios de clase propuestos por los profesores y que se resuelven desde la información consultada o desde el propio discurso de los maestros. En otros casos, las situaciones problemáticas derivadas de los contextos local o institucional se abordan desde discursos cotidianos de los estudiantes, donde cobran poca relevancia elementos de carácter conceptual.

El desempeño 49 es el único entre los 65 que no es propiciado por ninguno de los profesores. Es importante generar ambientes de aprendizaje que propendan al desarrollo de pensamiento hipotético y actividades de carácter experimental que permitan interrelacionar variables, así como el establecimiento de condiciones, relaciones y regularidades para un conjunto de eventos o situaciones complejas.

\section{Competencias científicas de pensamiento reflexivo y crítico}

Los desempeños del nivel inicial asociados a esta competencia, son promovidos por la mayoría de los profesores; sin embargo, se plantea la necesidad de que el maestro reflexione sobre la relación que existe entre las actividades propuestas y el alcance logrado respecto al desarrollo de los desempeños propios de esta competencia, y la permanencia de estos logros más allá del referente evaluativo.

En relación con los desempeños del nivel intermedio, los docentes promueven de manera general los desempeños 55 y 56; por otra parte, son contados los maestros que desarrollan el desempeño relacionado con emplear conceptos matemáticos para la construcción de significados, excepto en algunas clases de física y química, lo que reitera que las clases de ciencias naturales (biología) se asumen de una manera descriptiva, mientras que las clases de física y química en general se desarrollan desde una postura más explicativa.

La toma de decisiones y la elaboración de inferencias le permiten al estudiante construir y reconstruir significados, y en consecuencia, asumir una mirada particular y crítica respecto a la ciencia.

En lo que tiene que ver con el nivel avanzado, la mayoría de los maestros desarrollan los desempeños 60 y 61 , en tanto que 62, 63 y 65 los propician pocos profesores. 


\section{Conclusiones}

La investigación generó, a través de permanentes búsquedas y construcciones colectivas, un acercamiento metodológico particular para acceder al pensamiento y prácticas educativas de los maestros participantes, el cual se constituyó en conocimiento nuevo que enriqueció el saber práctico del equipo de investigación y de los docentes participantes. Este conocimiento se ofrece a la comunidad académica para su discusión y crítica. Por otra parte, en la metodología empleada se incluyeron la elaboración y la revisión de instrumentos para la producción y sistematización de los hallazgos, de los cuales destacamos las matrices, que se han utilizado a su vez como pautas de logros deseables en la enseñanza de las ciencias experimentales por docentes en servicio.

El trabajo conjunto del equipo de investigación con los maestros participantes en torno a la revisión de las conceptualizaciones sobre competencias científicas y valores, además de ser un escenario para la actualización teórica, posibilitó no sólo lecturas particulares de estos marcos conceptuales desde el pensamiento y conocimiento profesional y experiencial de los maestros, sino que permitió realizar una reconstrucción teórica desde el sentido particular que los profesores participantes asignan a estos referentes.

\section{Bibliografía}

Arnal, J. et al. (1992). Investigación educativa fundamentos y metodologías. Barcelona: Editorial Labor.

Arteta, J.; Chona, G.; Fonseca, G.; Martínez, S.; e Ibáñez, S. (2002). Las competen-
Los maestros orientan en mayor proporción desempeños que corresponden a competencias científicas básicas de niveles inicial e intermedio, y las competencias científicas investigativas en el nivel inicial; en menor grado, se propician desempeños que corresponden a las competencias científicas de pensamiento reflexivo-crítico.

Respecto a las competencias básicas se puede concluir que los maestros desarrollan desempeños en los estudiantes, referidos al manejo de información en torno al contenido básico, en correspondencia con la imagen de conocimiento centrado en la información; en tal sentido, las frecuencias más altas se encuentran cuando el estudiante deberá decodificar y actuar en relación con lo establecido, desde el trabajo sobre los textos, desde su discurso y desde las guías, y por el contrario, son muy escasas las prácticas pedagógicas donde se privilegie el trabajo experimental.

Por último, se puede señalar que el desarrollo de estos desempeños permite iniciar en el estudiante el desarrollo de pensamiento científico, propósito propio de la enseñanza de las ciencias, que son factibles en tanto se generen ambientes de aprendizaje complejos que posibiliten la interconexión de elementos como la reflexión, autoconciencia, toma de decisiones, postura crítica y propositiva frente a su mundo natural y social. $\Delta$

cias científicas y el pensamiento de los profesores de ciencias naturales. El oficio de investigar. Educación y pedagogía frente a nuevos retos, 3. Colección Desarrollos en Investigación 
en Educación. Bogotá: Universidad Pedagógica Nacional-CIUP.

Cárdenas, F. (1998). Desarrollo y evaluación de los procesos de razonamiento complejo en ciencias. Revista TEA, 3, Bogotá: Universidad Pedagógica Nacional.

Cárdenas, F. (1999). Conocimientos, logros, habilidades, competencias y... ¿Qué evaluar? Hacia una cultura de la evaluación para el siglo XXI. Bogotá: Universidad Nacional de Colombia.

Cárdenas, F. y Sarmiento, F. (2000). Desarrollo y evaluación de competencias en ciencias. Proyecto pedagógico y competencias. Bogotá: Universidad Nacional de Colombia.

Carr, W. y Kemis, S. (1988). Teoría crítica de la enseñanza. Barcelona: Martínez Roca.

Chomsky, N. (1971). El lenguaje y el entendimiento. Biblioteca Breve Seix Barral.

Chona, G.; Castaño, N.; Arteta, J.; Leudo, M.; Valencia S. y Martínez, S. (1998). Aproximación a las creencias que orientan la práctica del profesor de biología. Revista TEA, 4, Bogotá: Universidad Pedagógica Nacional.

Chona, G.; Arteta, J.; y Martínez, S. (2001). El pensamiento educativo implícito en las prácticas de enseñanza de la biología. Revista TEA, 10. Bogotá: Universidad Pedagógica Nacional.

Chona G., et al. (2004). Informe final investigación competencias científicas y formación en valores. Un estudio desde el pensamiento de profesores de ciencias experimentales. Bogotá: Universidad Pedagógica NacionalCIUP.

Delors J. (1996). La educación encierra un tesoro. Informe a la Unesco de la Comisión Internacional sobre la Edu- cación para el siglo XXI. Santillana, Ediciones Unesco.

Díaz, M. (2002). En: G. Bustamante, S. de Zubiría, M. Bacarat; N. Graciano, L. Marín, J. Gómez, E. Serrano. (2002). El concepto de competencia. Una mirada interdisciplinar, II. Bogotá: Sociedad Colombiana de Pedagogía- Alejandría Libros.

Escobedo, H. (2001). Desarrollo de competencias básicas para pensar científicamente. Una propuesta didáctica para ciencias naturales. Bogotá: Colciencias.

Gallego, M. J. (1991). Investigación sobre pensamientos del profesor: aproximaciones al estudio de las teorías y creencias de los profesores. Revista Española de Pedagogía, 189.

Gardner, H. (1993). La mente no escolarizada. Cómo piensan los niños y cómo deberían enseñar las escuelas. Barcelona: Paidós.

Hymes, D. (1972). Acerca de la competencia comunicativa. Forma y Función, 9, (Juan Gómez, trad.) (1996).

Marcelo, C (2005). La investigación sobre el conocimiento de los profesores y el proceso de aprender a enseñar. Una revisión personal. En: G. Perafán y A. Adúriz-Bravo (comps.) (2002). Pensamiento y conocimiento de los profesores. Debate y perspectivas internacionales. Bogotá: Universidad Pedagógica Nacional-Colciencias.

Ministerio de Educación Nacional (1994). Ley 115. Ley General de educación. Colombia.

Ministerio de Educación Nacional (1998). Lineamientos curriculares. Ciencias naturales y educación ambiental. Bogotá: Cooperativa Editorial Magisterio. 
Ministerio de Educación Nacional. (2002). Estándares para la excelencia en la educación. Estándares para las áreas de matemáticas, lengua castellana y ciencias naturales y educación ambiental para la educación preescolar, básica y media. Bogotá.

Ministerio de Educación Nacional. Instituto Colombiano para el Fomento de la Educación Superior (1999). Examen de Estado para el ingreso a la educación superior. Cambios para el siglo XXI. Propuesta general. Bogotá.

Misión Ciencia, Educación y Desarrollo (1995). Colombia, al filo de la oportunidad. Bogotá: Idep.

Perafán, G. y Adúriz-Bravo, A. (comps.) (2002). Pensamiento y conocimiento de los profesores. Debate y perspectivas internacionales. Bogotá: Universidad Pedagógica Nacional-Colciencias.

Perkins D. (1998). ¿Qué es la comprensión? En M. Stone (comp.) (1999). La enseñanza para la comprensión. Vinculación entre la investigación y la práctica. Buenos Aires: Paidós.

Porlán, R.; Rivero, A.; y Martín del Pozo, R. (1997). Conocimiento profesional y epistemología de los profesores, I: Teoría, métodos e instrumentos. Enseñanza de ciencias, 15, 2.
Reyes, L. y Salcedo, L. (1998). Acciones de maestros de ciencias: creencias, roles, metas y contextos en la enseñanza y el aprendizaje. Revista TE $\Delta$, 3, Bogotá: Universidad Pedagógica Nacional.

Reyes, L.; Salcedo L., y Perafán, G. (1999). Acciones y creencias. Tesoro oculto del educador, I. Bogotá: Universidad Pedagógica Nacional.

Shulman, L. S. (1989). Paradigmas y programas de investigación en el estudio de la enseñanza. Una perspectiva contemporánea. En: M. Wittrock (1989). La investigación en la enseñanza, I, Barcelona: Paidós.

Torrado, C. (1999). El desarrollo de las competencias: una propuesta para la educación colombiana. Hacia una cultura de la evaluación para el siglo XXI. Bogotá: Universidad Nacional de Colombia.

Wittrock, M. (1997). La investigación de la enseñanza. I, II, III. Barcelona: Paidós.

Yerrit, R.; Parke, L. y Nugent, J. (1997). En: L., Reyes, L., Salcedo y G. Perafán, (1999). Acciones y creencias, Tesoro oculto del educador. I, Bogotá: Universidad Pedagógica Nacional. 